\title{
CMEs driven by an eruptive prominence
}

\author{
G. P. Zhou ${ }^{1}$, J. X. Wang ${ }^{1}$, J. Zhang ${ }^{1}$, P. F. Chen ${ }^{2}$, H. S. Ji ${ }^{3}$, \\ and K. Dere ${ }^{4}$ \\ ${ }^{1}$ National Astronomical Observatories, Chinese Academy of Sciences, Beijing 100012, China \\ email: zhougp@ourstar.bao.ac.cn \\ ${ }^{2}$ Department of Astronomy, Nanjing University, Nanjing 210093, China \\ ${ }^{3}$ Purple Mountain Observatory, 2 Beijing Xi Lu, Nanjing 210008, China \\ ${ }^{4}$ George Mason University, 4400 University Drive, USA
}

\begin{abstract}
A clear case on Dec. 282002 is presented. It is about the initiation of two successive CMEs, which were related to an eruptive prominence. In EIT $304 \AA$ and $195 \AA$ observations, we found a long filament severely twisting in a piece of its fragments, which appeared as a prominence on Dec. 26. Then, the prominence converted its twist into writhe. Two days later, the prominence displayed a slow rising motion for hours. There happened internal twisting and mass motion before the prominence rapid acceleration and final eruption. Two successive CMEs recorded by LASCO C2 coronagraph corresponded to the early rising and the subsequently eruptive phases of the prominence, respectively. Evidence of magnetic reconnection, i.e., a cusp structure and post-flare loops in EUV wavebands, and hard X-ray sources in the corona, were observed after the prominence disruption. It appears that the kink instability and the mass drainage in the prominence played important roles in triggering two CMEs' initiation. We suspected that the rather impulsive acceleration of the second CME resulted from magnetic reconnection beneath the prominence.
\end{abstract}

Keywords. coronal mass ejections, prominence.

\section{Observations}

The eruption process of this event was observed by EIT at $304 \AA$ and $195 \AA$ Tं he LASCO observations are used to view the associated CMEs. Additionally, the observations from GOES soft X-ray (SXR) and RHESSI hard X-ray (HXR) are also used to study the highenergy process of this event. Time sequence of the CME initiation is listed in Table 1 . In Figure 1b, two lines forming an angle of significant magnitude indicate the same leg of the arcade envelope at different time of 11:48 UT (dashed) and 13:48 UT (solid), respectively. The arrow in Figures 1c and 1d denotes the material motion along the prominence legs. In order to show the mass drainage more clearly, Figures $1 \mathrm{c}$ and $1 \mathrm{~d}$ are presented with a method to make edge enhancement for the images. Two dashed curves in Figures 1e and If denote the outlines of the adjacent arcades. A cusp shape structure (Fig. 1f) appears after the prominence disruption.

\section{Conclusions}

We described the observed scenario of this event as four evolving phases, which are schematically shown in Figure 2. (1) Before being disturbed, a fragment of the long filament presented strong twist and was overlaid by multiple-layer arcades at different heights (Fig. 2a). The prominence twisted more than one turn, which exceeded a critical value for the kink instability Hood \& Priset (1979). (2) As the kink instability developing, the prominence rose and converted part of its twists to writhes, and the prominence and 
Table 1. Time sequences of CME initiation

\begin{tabular}{|c|c|c|}
\hline Date & Time $(U T)$ & Phenomena \\
\hline 25 & $00: 36$ & a piece of the long filament began to rise \\
\hline 25 & 01:19 & the twist of the prominence was discernable in EIT $195 \AA$ images \\
\hline 27 & 01:19 & the prominence began to writhe \\
\hline 28 & $11: 48$ & the apex of the prominence began to brighten in $195 \AA$ images \\
\hline - & $11: 48 \sim 13: 48$ & $\begin{array}{l}\text { the prominence is slowly ascending, untwisting, } \\
\text { and expanding continuously }\end{array}$ \\
\hline- & $13: 48 \sim 14: 36$ & reactivation phase of the prominence before its catastrophic eruption \\
\hline - & $13: 54$ & the first CME appeared in LASCO C2 images \\
\hline - & $14: 36 \sim$ & the prominence disrupted \\
\hline - & $15: 24$ & cusp shape structure first appeared \\
\hline - & $16: 30$ & the second associated CME appeared \\
\hline - & $16: 48$ & the apex part of the prominence ran out of the EIT field of view \\
\hline - & $17: 30$ & the core of the second CME first appears \\
\hline - & $18: 12$ & post-flare loop first appeared \\
\hline - & $18: 50 \sim 19: 11$ & weak HXR source appeared on the top of post-fare loops \\
\hline
\end{tabular}

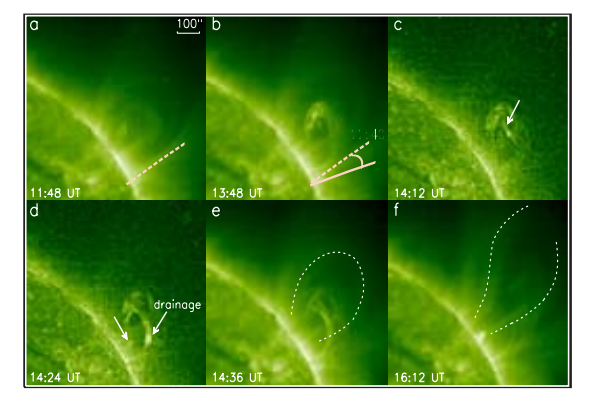

Figure 1. EIT $195 \AA$ images show the prominence disruption with the combination of kink and drainage instabilities.

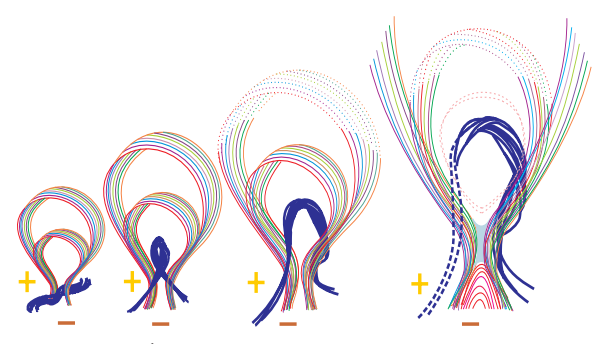

(d)

Figure 2. A cartoon demonstrating the scenario of the event.

its arcades reached a new height (Fig. 2b). (3) When the prominence was rising under a kink instability, the plasma in the prominence might drain back to the solar surface along the deformed field lines (Fig. 2c), which produced magnetic buoyancy force to drive the prominence rising again. As a consequence, the eruptive prominence impelled its overlaying arcade envelope ascending to form the first slow CME in LASCO C2 field of view. (4) The prominence re-activated, manifested by its internal twist and mass motion for about 40 minutes. Then the prominence disrupted. Its adjacent arcades were driven and restructured to become the second CME. The reconnection at the current sheet (see the light solid area in Fig. 2d) in the wake of erupted prominence led to an additional drastic acceleration of the prominence and its overlaying arcades for the second CME.

\section{Acknowledgements}

The work is supported by the NNSF of China (G10573025, G10233050, and G4050421) and the NKBRSF (G2000078404).

\section{Reference}

Hood, A. W., \& Priset, E. R. 1979, Sol. Phys., 64, 303 